\title{
GENERALIZED MATRIX ALGEBRAS
}

\author{
W. P. BROWN
}

1. Introduction. The algebras considered here arose in the investigation of an algebra connected with the orthogonal group. ${ }^{1}$ We consider an algebra $\mathfrak{A}$ of dimension $m n$ over a field $K$ of characteristic zero, and possessing a basis $\left\{e_{i j}\right\} \quad(1 \leqslant i \leqslant m ; 1 \leqslant j \leqslant n)$ with the multiplication property

$$
e_{i j} e_{p q}=\phi_{j p} e_{i q}
$$

$\phi_{j p} \in K$.

The field elements $\phi_{i j}$ form a matrix $\Phi=\left(\phi_{i j}\right)$ of order $n \times m$. It will be called the multiplication matrix of the algebra relative to the basis $\left\{e_{i j}\right\}$.

Such algebras will be called generalized matrix algebras. If $m=n$ and $\phi_{i j}=$ $\delta_{i j}$ (the Kronecker delta) we have a total matrix algebra.

An element $b$ of $\mathfrak{A}$ has an expression in terms of the basis $\left\{e_{i j}\right\}$ of the form

$$
b=\sum_{i=1}^{m} \sum_{j=1}^{n} b_{i j} e_{i j}
$$$$
b_{i j} \in K
$$

The correspondence $b \rightarrow B=\left(b_{i j}\right)$ is a one to one correspondence between the elements of $\mathfrak{A}$ and the set of $m \times n$ matrices over $K$. If $a \in \mathfrak{A}$ and $a \rightarrow$ $A=\left(a_{i j}\right)$, then

$$
\begin{aligned}
a b & =\sum_{i, j} a_{i j} e_{i j} \sum_{p, q} b_{p q} e_{p q} \\
& =\sum_{i, q}\left(\sum_{j, p} a_{i j} \phi_{j p} b_{p q}\right) e_{i q} .
\end{aligned}
$$

It follows that the product $a b$ corresponds to the matrix $A \Phi B ; a b \rightarrow A \Phi B$. The most general change of basis of $\mathfrak{A}$ may be effected by a transformation of the type

$$
f_{i j}=\sum_{\lambda=1}^{m} \sum_{\mu=1}^{n} \sigma_{i j}^{\lambda \mu} e_{\lambda \mu}
$$$$
\sigma_{i j}{ }^{\lambda \mu} \in K
$$

$(1 \leqslant i \leqslant m ; 1 \leqslant j \leqslant n)$. We use double suffix notation with lexicographic ordering to describe the matrix of this transformation. The element of its $(i j)$ th row and $(\lambda \mu)$ th column is $\sigma_{i j}{ }^{\lambda \mu}$. The elements $f_{i j}$ of $\mathfrak{A}$ constitute a basis for $\mathfrak{A}$ if and only if this matrix is non-singular. We consider however a special type of transformation, namely:

$$
f_{i j}=\sum_{\lambda=1}^{m} s_{i \lambda} e_{\lambda j}
$$$$
s_{i \lambda} \in K
$$

This may be written in the form of (2) by setting $\sigma_{i j}{ }^{\lambda \mu}=s_{i \lambda} \delta_{j \mu}$. This is the element of the $(i j)$ th row and $(\lambda \mu)$ th column of the Kronecker product $S \times I$ where $S$ is the $m \times m$ matrix $\left(s_{i j}\right)$ and $I$ is the $n \times n$ unit matrix. It follows

Received November 2, 1953.

${ }^{1}$ The algebra concerned is $\omega_{f}^{n}$. For definition see (1) and (2, chap. V, 5). 
that the elements $f_{i j}$ in (3) constitute a basis for $\mathfrak{A}$, if and only if $S=\left(s_{i j}\right)$. is non-singular.

The nature of the multiplication rule is preserved under transformations of the type occurring in (3). Indeed

$$
\begin{aligned}
f_{i j} f_{p q} & =\sum_{\lambda} s_{i \lambda} e_{\lambda j} \sum_{\mu} s_{p \mu} e_{\mu q} \\
& =\sum_{\lambda, \mu} s_{i \lambda} s_{p \mu} \phi_{j \mu} e_{\lambda q} \\
& =\left(\sum_{\mu} s_{p \mu} \phi_{j \mu}\right) f_{i q},
\end{aligned}
$$

by (3). Hence $f_{i j} f_{p q}=\psi_{j p} f_{i q}$ where $\psi_{j p}=\sum_{\mu} \phi_{j \mu} s_{p \mu}$. Relative to the new basis $\left\{f_{i j}\right\}$, the algebra has therefore the multiplication matrix $\Psi=\Phi S^{T}$, where $S^{T}$ denotes the transpose of $S$.

We make a further change of basis, namely

$$
g_{i j}=\sum_{\lambda=1}^{n} f_{i \lambda} r_{\lambda j}, \quad \quad r_{\lambda j} \in K,
$$

$(1 \leqslant i \leqslant m ; 1 \leqslant j \leqslant n)$. Again the elements $g_{i j}$ of $\mathfrak{A}$ constitute a basis if and only if the $n \times n$ matrix $R=\left(r_{i j}\right)$ is non-singular. The multiplication rule is again transformed;

$$
g_{i j} g_{p q}=\theta_{j p} g_{i q} \text { where } \theta_{j p}=\sum_{\lambda} r_{\lambda j} \psi_{\lambda p} \in K .
$$

Relative to the basis $\left\{g_{i j}\right\}$ the algebra has the matrix $\theta=R^{T} \Phi S^{T}$.

If $\Phi$ has rank $r$, non-singular matrices $R$ and $S$ may be chosen so that the $n \times m$ matrix $\theta$ is

$$
\Theta=\left(\begin{array}{ll}
I_{r} & 0 \\
0 & 0
\end{array}\right),
$$

where $I_{r}$ is the $r \times r$ unit matrix and all other submatrices of $\Theta$ are zero. A basis such as $\left\{g_{i j}\right\}$, relative to which the multiplication matrix has this simple form, will be called a special basis. While the types of transformation used preserve the rank of the multiplication matrix, it has not yet been demonstrated that the rank of a multiplication matrix is an invariant for $\mathfrak{A}$. This, however, will be obvious later.

2. The structure of generalized matrix algebras. We now assume that a special basis has been chosen for $\mathfrak{A}$ and that the multiplication matrix $\Phi$ has the special form of $\Theta$ above and has rank $r$. Suppose that an element $b \in \mathfrak{A}$ has a matrix $B$ whose partitioned form is

$$
B=\left(\begin{array}{ll}
B_{1} & B_{2} \\
B_{3} & B_{4}
\end{array}\right),
$$

the dimensions of the submatrices being $r \times r$ for $B_{1}, r \times n-r$ for $B_{2}$, $m-r \times r$ for $B_{3}$ and $m-r \times n-r$ for $B_{4}$.

The radical of $A$ consists of all elements that are properly nilpotent, i.e. all elements $b$ of $\mathfrak{A}$ such that for every $a \in \mathfrak{A}, a b$ is nilpotent. The matrix cor- 
responding to the $t$ th power of $b$ is $(B \Phi)^{t-1} B$. It follows that $b$ is nilpotent if and only if $B \Phi$ is a nilpotent matrix.

Let $a$ and $c$ be elements of $\mathfrak{A}$ whose matrices are $A$ and $C$ respectively. The matrix of the product $a b c$ is the product of matrices $A \Phi B \Phi C$. Now suppose $B_{1}=0$. Then

$$
\Phi B \Phi=\left(\begin{array}{ll}
I_{r} & 0 \\
0 & 0
\end{array}\right)\left(\begin{array}{ll}
0 & B_{2} \\
B_{3} & B_{4}
\end{array}\right)\left(\begin{array}{ll}
I_{r} & 0 \\
0 & 0
\end{array}\right)=0 .
$$

It follows that $b$ is properly nilpotent and has index of nilpotence $\leqslant 3$.

On the other hand, for $b$ to be properly nilpotent it is necessary that the matrix $A \Phi B \Phi$ be nilpotent for all matrices $A$.

$$
A \Phi B \Phi=\left(\begin{array}{ll}
A_{1} & A_{2} \\
A_{3} & A_{4}
\end{array}\right)\left(\begin{array}{ll}
I_{r} & 0 \\
0 & 0
\end{array}\right)\left(\begin{array}{ll}
B_{1} & B_{2} \\
B_{3} & B_{4}
\end{array}\right)\left(\begin{array}{ll}
I_{r} & 0 \\
0 & 0
\end{array}\right)=\left(\begin{array}{ll}
A_{1} B_{1} & 0 \\
A_{3} B_{1} & 0
\end{array}\right)
$$

The $t$ th power of this matrix has $\left(A_{1} B_{1}\right)^{t}$ in the first submatrix position. It follows that $b$ is properly nilpotent only if $A_{1} B_{1}$ is nilpotent for all $r \times r$ matrices $A_{1}$. This can only occur if $B_{1}=0$. Hence the radical of $\mathfrak{A}$ consists of all those elements $b$ whose matrices relative to a special basis, have their first submatrix zero.

A Wedderburn decomposition of $\mathfrak{A}$ into a direct sum of a semisimple subalgebra $\mathfrak{B}$ and the radical $\mathfrak{N}$ is now clear. $\mathfrak{B}$ consists of all elements $b$ of $\mathfrak{A}$ whose matrices relative to a special basis, have the submatrices $B_{2}, B_{3}$ and $B_{4}$ all zero. For such elements the mapping $b \rightarrow B_{1}$ is a ring isomorphism of $\mathfrak{B}$ onto the total matrix algebra of degree $r$ over $K$. Hence $\mathfrak{B}$ is simple and $r$ is an invariant of the algebra.

We see that a generalized matrix algebra is either simple $(m=n=\operatorname{rank} \Phi)$ or is non semisimple and simple modulo its radical. If the algebra is simple it certainly possesses an identity element. On the other hand let the generalized matrix algebra $\mathfrak{A}$ possess an identity element $e$ so that $e a=a e=a$ for all $a$ in $\mathfrak{A}$. Let $E$ and $A$ be the corresponding matrices. We must have $E \Phi A=$ $A \Phi E=A$ for all matrices $A$. Hence $E \Phi$ must be the $m \times m$ unit matrix and $\Phi E$ must be the $n \times n$ unit matrix. This can happen only if $m=n$ and $\Phi$ is non-singular. The algebra is then simple. We restate the above result in a

Theorem. A generalized matrix algebra is either

(i) simple, or

(ii) non-semisimple and simple modulo its radical.

It is simple if and only if it possesses an identity element.

\section{REFERENCES}

1. R. Brauer, On algebras which are connected with the semisimple continuous groups, Ann. Math., 38 (1937), 857.

2. H. Weyl, The classical groups (Princeton, 1946).

King's College, Aberdeen 